\title{
Survey on Lehmer Problems
}

\author{
María Carrizosa
}

\begin{abstract}
We survey the known results and open questions related to the Lehmer Problem. We start by recalling the "classical" conjecture : the existence of a lower bound for the height of an algebraic number (not a root of unity) of the form $h(\alpha) \geq \frac{c}{[\mathbb{Q}(\alpha): \mathbb{Q}]}$ and the generalizations to higher dimension. We discuss afterwards, the "relative" versions of these problems, that is, replacing de degree of $\alpha$ over $\mathbb{Q}$ by the degree over $\mathbb{Q}^{\mathrm{ab}}$. We give the most recent results on these questions and we discuss briefly an application of the latest to the Pink-Zilber conjecture.
\end{abstract}

\section{Introduction}

In this section we recall some basic facts about projective and canonical heights.

Let $K / \mathbb{Q}$ be a number field and $\alpha$ a point in $\mathbb{P}^{n}(K)$. The absolute logarithmic height of $\alpha$ is

$$
h(\alpha)=\frac{1}{[K: \mathbb{Q}]} \sum_{v \in M_{K}} n_{v} \log \max \left\{\left|\alpha_{0}\right|_{v}, \ldots,\left|\alpha_{n}\right|_{v}\right\}
$$

where the sum runs over all the places of $K$ normalized in the usual way $\left(|p|_{v}=p^{-1}\right.$ for $\left.v \mid p\right), n_{v}$ denotes the local degree $\left(n_{v}=\left[K_{v}: \mathbb{Q}_{v}\right]\right)$ and $\left(\alpha_{0}, \ldots, \alpha_{n}\right)$ is some choice of coordinates of $\alpha$ such that $\alpha_{i} \in K$ for $i=$ $0, \ldots, n$. We can easily check from the definition, that the height does not depend on the choice of coordinates and is invariant under base field extension.

If $\alpha \in \mathbb{G}_{m}^{n}(\overline{\mathbb{Q}}) \hookrightarrow \mathbb{P}^{n}(\overline{\mathbb{Q}})$ we define a height in $\mathbb{G}_{m}^{n}$ by $h(\alpha)=h(1: \alpha)$.

Among the most important results about heights we have the following

Theorem 1.1 (Northcott). Let $C, D \in \mathbb{R}$. Then the set

$$
\left\{\alpha \in \mathbb{P}^{n}(\overline{\mathbb{Q}}), \quad[\mathbb{Q}(\alpha): \mathbb{Q}] \leq D, \quad h(\alpha) \leq C\right\}
$$

is finite. 
We can notice that $h(\alpha) \geq 0$ for all $\alpha \in \mathbb{P}^{n}(\overline{\mathbb{Q}})$ so we can ask ourselves when this function vanishes. The following theorem answers this question.

Theorem 1.2 (Kronecker). Let $\alpha \in \mathbb{P}^{n}(\overline{\mathbb{Q}})$; then $h(\alpha)=0$ if and only if there is a set $\left(\alpha_{0}, \ldots, \alpha_{n}\right)$ of projective coordinates of $\alpha$ such that for all $i$, $0 \leq i \leq n$ one has either $\alpha_{i}=0$ or $\alpha_{i}$ is a root of unity.

We can extend the definition of heights to points in algebraic varieties. This tool should permit to transform geometric facts into arithmetic relations. We refer to [HS00] and [BG06], part B, pp.168-242 for a complete construction of these heights and their properties. The main idea is the following : let $V$ be a projective variety defined over $\mathbb{Q}$ and a given morphism $\phi: V \rightarrow \mathbb{P}^{n}$. Then the height on $V$ relative to $\phi$ is the function

$$
\begin{aligned}
h_{\phi}: V(\mathbb{Q}) & \rightarrow[0, \infty) \\
P & \mapsto h(\phi(P))
\end{aligned}
$$

where $h$ is the projective height defined above. Using the relationship between divisors and embeddings, we can show that this definition depends only on the equivalence class of divisors (or line bundles) on $V$, up to a bounded function. This construction is due to Weil and we call it the Height Machine (following Silverman).

When $V$ is an abelian variety, a careful choice of a divisor gives us a height with nice properties with respect to the group law in $V$.

Proposition 1.3. Let $A / K$ be an abelian variety defined over a number field and $\mathcal{L}$ an ample, symmetric line bundle. Then there is a height function $h_{\mathcal{L}}$ with the following properties

(1) For each integer $m, \quad h_{\mathcal{L}}([m] P)=m^{2} h_{\mathcal{L}}(P)+O(1) \quad \forall P \in A(\bar{K})$.

(2) $h_{\mathcal{L}}(P+Q)+h_{\mathcal{L}}(P-Q)=2 h_{\mathcal{L}}(P)+2 h_{\mathcal{L}}(Q)+O(1) \quad \forall P, Q \in A(\bar{K})$.

(3) For any real numbers $h>0, d>0$, the set

$$
\left\{P \in A(\bar{K}) \mid h_{\mathcal{L}}(P) \leq h,[K(P): K] \leq d\right\}
$$

is finite.

Finally, we can construct from $h_{\mathcal{L}}$ a canonical height satisfying all the above properties for $O(1)=0$, and therefore giving a quadratic form on $A(\bar{K})$ (and so a finite-dimensional Euclidean vector space structure on $A(\bar{K}) \otimes \mathbb{R})$. This is done by the following formula :

$$
\hat{h}_{\mathcal{L}}(P)=\lim _{n \rightarrow+\infty} \frac{h_{\mathcal{L}}\left(\left[2^{n}\right] P\right)}{4^{n}} .
$$

In addition we have an analogue of the Kronecker theorem :

Theorem 1.4 (Néron-Tate). Let $A / K$ be an abelian variety defined over a number field and $\mathcal{L}$ an ample, symmetric line bundle. 
(1) $\hat{h}_{\mathcal{L}}(P)=h_{\mathcal{L}}(P)+O(1)$.

(2) $\hat{h}_{\mathcal{L}}(P) \geq 0$, with equality if and only if $P$ is a point of finite order.

Coming back to the $\mathbb{G}_{m}$ setting, once we know that the height does not vanish, how far can it go from zero?

We can check that for every strictly positive integer $m, h\left(\alpha^{m}\right)=|m| h(\alpha)$. Looking for example at $(\sqrt[m]{2})$ for large $m$ we see that if the degree of $\alpha$ goes to infinity, from the above property, the height goes to zero. We could then expect some bound depending on the degree of $\alpha$ over $\mathbb{Q}$. This leads us to the Lehmer Problem :

Conjecture 1.5. There exists a real number $c>0$ such that for every $\alpha \in \overline{\mathbb{Q}}^{*}$ such that $\alpha$ is not a root of unity, then

$$
h(\alpha) \geq \frac{c}{[\mathbb{Q}(\alpha): \mathbb{Q}]} .
$$

In some particular cases, it has being proved that this conjecture is true. This is the reason that nowadays we state it in this form, but Lehmer was not affirmative in his paper [Leh33]. He formulated the question the other way around and added "whether this is true or not, we do not know". To be more precise, Lehmer was searching for large primes and formulated this question in terms of the Mahler measure of $\alpha, \operatorname{e.g} \exp ([\mathbb{Q}(\alpha): \mathbb{Q}] h(\alpha))$.

\section{Classical Lehmer Problem}

In this section we explain the known results around the "classical Lehmer problem" stated above. We start with dimension 1 and explain the best possible conjectures in higher dimension.

First of all, we notice that conjecture 1.5 is true for an algebraic number that is not an algebraic integer. In fact, we can easily show that if $\alpha_{1}, \ldots, \alpha_{d}$ are the conjugates of $\alpha$ over $\mathbb{Q}$ and $a_{0}$ is the leading coefficient of its minimal polynomial, then

$$
h(\alpha)=\frac{1}{d} \sum_{i=1}^{d} \log \max \left\{1,\left|\alpha_{i}\right|\right\}+\frac{1}{d} \log \left|a_{0}\right|,
$$

and so if $h(\alpha)<\frac{\log 2}{d}$ then $a_{0}=1$, that is, $\alpha$ is an algebraic integer.

Another important result is due to Smyth [Smy71] who proved that if $\alpha^{-1}$ is not in the Galois orbit of $\alpha$, then conjecture 1.5 is true for $\alpha$. Other cases have confirmed that bound 1.5 or even better bounds can be true. See for example [CNS77], [Mig78], [Rob65]. 
The best known result in the way of proving conjecture 1.5 is a theorem by Dobrowolski [Dob79] :

Theorem 2.1. There is a positive constant $c$, such that for all $\alpha \in \overline{\mathbb{Q}}^{*}$ not a root of unity, we have

$$
h(\alpha) \geq \frac{c}{D}\left(\frac{\log \log 3 D}{\log 2 D}\right)^{3}
$$

where $D=[\mathbb{Q}(\alpha): \mathbb{Q}]$.

Dobrowolski showed that we can take $c=\frac{1}{1200}$. Some refinements for the numerical constant where found by other authors, among them Voutier [Vou96], who showed that we can take $c=\frac{1}{4}$ if $D \geq 2$. Until now, any improvement has been done in the degree part of the bound.

Conjecture 1.5 has a natural statement in the context of elliptic curves.

Conjecture 2.2. Let $E / K$ be an elliptic curve over a number field $K$ and $\hat{h}$ the Néron-Tate height on $E(\bar{K})$. There is a positive constant $c(E / K)$ such that for all $P \in E(\bar{K}) \backslash E_{\text {tors }}$ we have

$$
\hat{h}(P) \geq \frac{c(E, K)}{[K(P): K]} .
$$

Laurent [Lau83] proved an "up to epsilon" version of this conjecture under the hypothesis that $E$ has complex multiplication. This follows from the strategy of Dobrowolski for proving theorem 2.1; in fact, his idea was to extrapolate on ultrametric places using Fermat's little theorem. To do this in the setting of an elliptic curve, we have to be able to lift the Frobenius map in characteristic 0 for a large amount of primes. That explains the need for the CM hypothesis. We remark that we could replace this hypothesis by the Lang-Trotter conjecture for elliptic curves.

Theorem 2.3 (Laurent). Let $E / K$ be an elliptic curve with complex multiplication over a number field $K$. Then there is a positive constant $c(E / K)$ such that for every point $P \in E(\bar{K}) \backslash E_{\text {tors }}$ we have

$$
\hat{h}(P) \geq \frac{c(E / K)}{D}\left(\frac{\log \log 3 D}{\log 2 D}\right)^{3}
$$

where $D=[K(P): K]$.

Generalisations to higher dimension have been largely studied : by David and Hindry [DH00] for abelian varieties and Amoroso and David [AD99] for points on $\mathbb{G}_{m}^{n}$.

Before explaining their results, we briefly recall the definition of the degree of a variety. 
Let $X$ a proper variety of dimension $n$ defined over $K, \mathcal{L}$ a line bundle (or equivalently an invertible sheaf or Cartier divisor) over $X$ and $V$ a subvariety of $X$ of dimension $k$. Let $D$ be the Cartier divisor associated to $\mathcal{L}$. We can construct an intersection product denoted by

$$
D \cdot V
$$

that gives a $(k-1)$-cycle supported on $|D| \cap V$ (see [Ful84] chap.I and chap.II).

Definition 2.4. Let $\alpha=\sum n_{P}[P]$ a 0-cycle over $X$. We define the degree of the 0-cycle $\alpha$ by :

$$
\operatorname{deg} \alpha=\sum n_{P}[K(P): K]
$$

We define the degree of a variety $V$ with respect of $\mathcal{L}$ by the degree of the o-cycle

$$
\underbrace{D \cdots D}_{k \text { times }} \cdot V
$$

For example, if $V=\{x\}$ for $x \in X(\bar{K})$, the degree $\operatorname{deg}_{\mathcal{L}}(V)$ is equal to $[K(x): K]$. If $V$ is an hypersurface defined by an irreducible polynomial of degree $D$ over $K$, then the degree of $V$ is $D$.

As we will see, in higher dimension there is a better invariant than the degree of a point : it is the so called obstruction index. There are many almost equivalent formulations for it, we'll use the following :

Definition 2.5. Let $X$ be an abelian variety defined over $K$ or a multiplicative torus $\mathbb{G}_{m}^{n}, \mathcal{L}$ a line bundle over $X$. For any $x \in X(\bar{K})$ we define

$$
\omega_{K}(x)=\min \left\{\operatorname{deg}_{\mathcal{L}}(V)^{\frac{1}{\operatorname{codim} V}}\right\}
$$

where $V$ runs over all subvarieties of $X$ defined over $K$ containing $x$.

We are now ready to state the higher dimension versions of the classical Lehmer Problem.

The best conjectural statement that we can expect in the multiplicative case is the following :

Conjecture 2.6. For any integer $n \geq 1$, there is a real number $c(n)>0$ such that for all $\alpha \in \mathbb{G}_{m}^{n}(\overline{\mathbb{Q}})$ whose coordinates are multiplicatively independent, we have

$$
h(\alpha) \geq \frac{c(n)}{\omega_{K}(\alpha)}
$$

We first notice that

$$
1 \leq \omega_{\mathbb{Q}}(\alpha) \leq n[\mathbb{Q}(\alpha): \mathbb{Q}]^{1 / n} .
$$


To see that this conjecture is the best we can expect, we can look at a point $\beta=\left(\beta_{1}, \ldots, \beta_{n}\right)$ and set $\alpha=\beta^{m}$ for $m \geq 1$. We then get $h(\beta)=\frac{1}{m} h(\alpha)$ and $\delta(\beta) \leq m \delta(\alpha)$. To see that the hypothesis on the coordinates of $\alpha$ is necessary, we can for look at the point $\alpha=(\sqrt[m]{2}, \ldots, \sqrt[m]{2})$. The "up to epsilon" version of this conjecture is the following theorem :

Theorem 2.7 (Amoroso-David). For any integer $n \geq 1$, there is a real number $c(n)>0$ such that for all $\alpha \in \mathbb{G}_{m}^{n}(\overline{\mathbb{Q}})$ whose coordinates are multiplicatively independent, we have

$$
h(\alpha) \geq \frac{c(n)}{\omega_{K}(\alpha)} \log \left(3 \omega_{K}(\alpha)\right)^{-\kappa(n)},
$$

where $\kappa(n)=(n+1)(n+1) !^{n}-n$.

In the abelian case we have the equivalent statement, known as "Abelian Lehmer Problem" :

Conjecture 2.8. Let $A / K$ an abelian variety of dimension $g$ over a number field $K$ and $\mathcal{L}$ an ample, symmetric line bundle over $A$. There is a real number $c(A, K, \mathcal{L})>0$ such that for every point $P \in A(\bar{K})$ of infinite order modulo every proper abelian subvariety of $A$, we have

$$
\hat{h}_{\mathcal{L}}(P) \geq \frac{c(A, K, \mathcal{L})}{\omega_{K}(P)} .
$$

As in the multiplicative version we can easily see that this is the best possible conjecture.

David and Hindry [DH00] proved this conjecture "up to epsilon" but unfortunately, as in the elliptic case, they had to impose a CM hypothesis on $A$ to lift the Frobenius in characteristic 0 to an endomorphism.

Theorem 2.9 (David-Hindry). Let $A / K$ an abelian variety of dimension $g$ with complex multiplication over a number field $K$ and $\mathcal{L}$ an ample, symmetric line bundle over $A$. There is a real number $c(A, K, \mathcal{L})>0$ such that for every point $P \in A(\bar{K})$ of infinite order modulo every proper abelian sub-variety of $A$, we have

$$
\hat{h}_{\mathcal{L}}(P) \geq \frac{c(A, K, \mathcal{L})}{\omega_{K}(P)}\left(\frac{\log \log 3 \delta_{\mathcal{L}}(P)}{\log \delta_{\mathcal{L}}(P)}\right)^{\kappa(g)}
$$

where $\kappa(g)=(2 g(g+1) !)^{g+2}$.

We conclude this section recalling that in the general case the best known result is a theorem by Masser [Mas], [Mas89]. He found that for $P \in A(\bar{K})$ of infinite order we have

$$
\hat{h}_{\mathcal{L}}(P) \geq c(A, K, \mathcal{L}) D^{-2 g-1} \log (2 D)^{-2 g}
$$

where $D=[K(P): K]$. 


\section{Relative Lehmer Problem}

We have seen that the classical Lehmer problem is true in some particular cases. A very important one is when an algebraic number $\alpha$ is defined over an abelian extension of $\mathbb{Q}$. In this direction Amoroso and Dvornicich [AD00] found an absolute bound for the height of such an element :

Theorem 3.1 (Amoroso-Dvornicich). For any $\alpha \in \mathbb{Q}^{\mathrm{ab}}$ that is not a root of unity,

$$
h(\alpha) \geq \frac{\log 5}{12} .
$$

The analogue of this result for abelian varieties was given by Baker and Silverman [Bak03], [Sil04], [BS04].

Theorem 3.2 (Baker-Silverman). Let $A / K$ be an abelian variety defined over a number field $K$. Let $\mathcal{L}$ be a symmetric ample line bundle on $A / K$ and let $\hat{h}: A(\bar{K}) \rightarrow \mathbb{R}$ be the associated canonical height. Then there is a constant $c(A, K, \mathcal{L})>0$ such that

$$
\hat{h}(P) \geq c(A, K, \mathcal{L}) \quad \text { for all nontorsion points } P \in A\left(K^{\mathrm{ab}}\right) .
$$

The next step is to generalize both the classical Lehmer Problem and these particular cases to obtain a stronger conjecture: the "relative Lehmer Problem". The idea is to replace (in the simplest setting) the degree of $\alpha$ over $\mathbb{Q}$ by the degree over the abelian closure of $\mathbb{Q}$. The first result in this direction is a theorem of Amoroso and Zannier [AZ00]

Theorem 3.3 (Amoroso-Zannier). Let $K$ be a number field. There is a constant $c(K)>0$ such that for every $\alpha \in \mathbb{G}_{m}(\bar{K})$ that is not a root of unity, then

$$
h(\alpha) \geq \frac{c(K)}{D}\left(\frac{\log \log 5 D}{\log 2 D}\right)^{13},
$$

where $D=\left[K^{\mathrm{ab}}(\alpha): K^{\mathrm{ab}}\right]$.

The relative version of theorem 2.3 was given by Ratazzi [Rat04] :

Theorem 3.4 (Ratazzi). Let $E / K$ an elliptic curve with complex multiplication over a number field $K$. Then there is a constant $c(E, K)>0$ such that for any $P \in E(\bar{K}) \backslash E_{\text {tors }}$ we have

$$
\hat{h}(P) \geq \frac{c(E, K)}{D}\left(\frac{\log \log 5 D}{\log 2 D}\right)^{13}
$$

where $D=\left[K^{\mathrm{ab}}(P): K^{\mathrm{ab}}\right]$. 
As in the classical setting, we can conjecture higher dimension versions of the relative Lehmer problem. We will state these for an abelian variety, the reader will easily imagine the multiplicative counterpart. The best possible conjecture in the general setting is the following :

Conjecture 3.5 (David). Let $A / K$ be an abelian variety of dimension $g$ defined over number field $K$, and $\mathcal{L}$ a symmetric ample line bundle on $A / K$. Then there is a constant $c(A, \mathcal{L}, K)>0$ such that for any $P \in A(\bar{K})$ that is not contained in a translate by a torsion point of a proper abelian subvariety of $A$, we have

$$
\hat{h}_{\mathcal{L}}(P) \geq c D^{-\frac{1}{g}}
$$

where $D=\left[K_{\text {tors }}(P): K_{\text {tors }}\right]$ and $K_{\text {tors }}=K\left(A_{\text {tors }}\right)$.

Before any result around this conjecture was known, Rémond proved in [Rém05] that such a conjecture implies a particular case of the Zilber-Pink conjecture. We'll explain this very briefly :

Theorem 3.6 (Rémond). Let $A$ be an abelian variety defined over $\bar{K}, X$ a transverse curve ${ }^{1}$ of $A$ and an integer $r \geq 2$. If conjecture 3.5 is true, then the set

$$
X^{[r]}=\bigcup_{\operatorname{codim} H \geq r} X \cap H(\bar{K})
$$

where $H$ runs over all algebraic subgroups of $A$ of codimension $\geq r$, is finite.

In fact, Rémond proved a more general result and showed that even an "up to epsilon" version of conjecture 3.5 was sufficient to prove this theorem.

This question can be considered as a generalization of the Manin-Mumford conjecture and was first solved by Bombieri, Masser and Zannier [BMZ99] for a curve in $\mathbb{G}_{m}^{n}$.

Roughly, the idea is to show in first place that the set $X^{[1]}$ is of bounded height. Then, using the Lehmer bound, one shows that the degree of a point in $X^{[2]}$ is bounded by an absolute constant. Northcott property permits to conclude that the set is then finite.

For CM elliptic curves, this question was solved by Viada [Via03] (we remark that in this case, theorem 2.3 was sufficient to prove this result).

We shall conclude by discussing the latest results around the "abelian relative Lehmer problem". In the multiplicative setting, Delsinne [Del07] has

\footnotetext{
${ }^{1}$ A curve $X$ is transverse in $A$ if it's not contained in any translate of a proper algebraic subgroup of $A$.
}

São Paulo J.Math.Sci. 3, 2 (2009), 317-327 
proved $^{2}$ the relative Lehmer problem "up-to epsilon" replacing the degree by the obstruction index (defined over $\mathbb{Q}^{\mathrm{ab}}$ ).

In the abelian context, we begin stating a stronger conjecture that implies all the previous ones. We'll need the following generalization of the obstruction index :

Definition 3.7. Let $X$ be an abelian variety defined over $K$ or a multiplicative torus $\mathbb{G}_{m}^{n}, \mathcal{L}$ a line bundle over $X$ and $V$ a subvariety of $X$ defined over an extension $F$ of $K$. For any $x \in X(\bar{K})$ we define

$$
\omega_{F}(x, V)=\min \left\{\left(\frac{\operatorname{deg}(Z)}{\operatorname{deg} V}\right)^{\frac{1}{\operatorname{codim}_{V}^{Z}}}\right\}
$$

where $Z$ runs over all subvarieties of $V$ defined over $F$ containing $x$.

Conjecture 3.8. Let $A / K$ be an abelian variety defined over number field $K$ and $\mathcal{L}$ a symmetric ample line bundle on $A / K$. Let $H$ be the smallest torsion subvariety ${ }^{3}$ of $A$ defined over $K$ containing $P$ and $H^{\circ}$ the $\bar{K}$ irreducible component of $H$ passing through 0 . Then, if $H^{\circ}$ is defined over $K$, there is a constant $c=c(g, K, \max \{1, h(A)\})>0$ such that for any $P \in A(\bar{K}) \backslash A(\bar{K})_{\text {tors }}$ we have

$$
\hat{h}_{\mathcal{L}}(P) \geq \frac{c}{\omega_{K}(P, H)}
$$

where .

We can easily see that this conjecture implies conjecture 2.8 (in that setting $\left.\omega_{K}(P, H)=\omega_{K}(P)\right)$. With some work, we can show that it also implies conjecture 3.5, and a strong version of it replacing the degree by the obstruction index. In [Car08] we prove the following :

Theorem 3.9. Under the hypothesis of conjecture 3.8, there is a constant $c(h(A), g, K)$ such that for every point $P \in A(\bar{K}) \backslash A_{\text {tors }}$ we have

$$
\hat{h}_{\mathcal{L}}(P) \geq \frac{c(h(A), g, K)}{\omega_{K}(P, H)}\left(\frac{\log \log \omega_{K_{\text {tors }}}(P, H) \operatorname{deg} H^{\circ}}{\log \omega_{K_{\text {tors }}}(P, H) \operatorname{deg} H^{\circ}}\right)^{\kappa\left(g_{0}\right)}
$$

where $g_{0}=\operatorname{dim} H$ and $\kappa\left(g_{0}\right)=2^{2 g_{0}+1}\left(g_{0}+1\right) !^{2 g_{0}} g_{0}^{2 g_{0}}$.

This gives an "up to epsilon" optimal version of conjecture 3.8 with an extra information about the polarization. As a consequence we have :

\footnotetext{
${ }^{2}$ In [Del07], Delsinne proves this result under a technical hypothesis. In a personal communication he explained that this hypothesis has been removed.

${ }^{3} \mathrm{~A}$ torsion subvariety is a union of translates of proper algebraic sub-groups by torsion points.
} 
Corollary 3.10. Let $A$ be an abelian variety with complex multiplication defined over $\bar{K}, X$ a transverse curve of $A$ and an integer $r \geq 2$. Then the set $A^{[r]}$ is finite.

\section{References}

[AD99] Francesco Amoroso and Sinnou David, Le problème de Lehmer en dimension supérieure, J. Reine Angew. Math. 513 (1999), 145-179.

[AD00] Francesco Amoroso and Roberto Dvornicich, A lower bound for the height in abelian extensions, J. Number Theory 80 (2000), no. 2, 260-272.

[AZ00] Francesco Amoroso and Umberto Zannier, A relative Dobrowolski lower bound over abelian extensions, Ann. Scuola Norm. Sup. Pisa Cl. Sci. (4) 29 (2000), no. 3, 711-727.

[Bak03] Matthew H. Baker, Lower bounds for the canonical height on elliptic curves over abelian extensions, Int. Math. Res. Not. (2003), no. 29, 1571-1589.

[BG06] Enrico Bombieri and Walter Gubler, Heights in Diophantine geometry, New Mathematical Monographs, vol. 4, Cambridge University Press, Cambridge, 2006.

[BMZ99] E. Bombieri, D. Masser, and U. Zannier, Intersecting a curve with algebraic subgroups of multiplicative groups, Internat. Math. Res. Notices (1999), no. 20, 1119-1140.

[BS04] Matthew H. Baker and Joseph H. Silverman, A lower bound for the canonical height on abelian varieties over abelian extensions, Math. Res. Lett. 11 (2004), no. 2-3, 377-396.

[Car08] María Carrizosa, Relative abelian lehmer problem, Ph.D. thesis, Université Paris et Marie Curie, 2008.

[CNS77] Thomas Callahan, Morris Newman, and Mark Sheingorn, Fields with large Kronecker constants, J. Number Theory 9 (1977), no. 2, 182-186.

[Del07] E. Delsinne, Autour du problème de Lehmer relatif dans un tore, Ph.D. thesis, Université de Caen/Basse Normandie, 2007.

[DH00] Sinnou David and Marc Hindry, Minoration de la hauteur de Néron-Tate sur les variétés abéliennes de type C. M, J. Reine Angew. Math. 529 (2000), $1-74$.

[Dob79] E. Dobrowolski, On a question of Lehmer and the number of irreducible factors of a polynomial, Acta Arith. 34 (1979), no. 4, 391-401.

[Ful84] William Fulton, Intersection theory, Ergebnisse der Mathematik und ihrer Grenzgebiete (3) [Results in Mathematics and Related Areas (3)], vol. 2, Springer-Verlag, Berlin, 1984.

[HS00] Marc Hindry and Joseph H. Silverman, Diophantine geometry, Graduate Texts in Mathematics, vol. 201, Springer-Verlag, New York, 2000, An introduction.

[Lau83] Michel Laurent, Minoration de la hauteur de Néron-Tate, Seminar on number theory, Paris 1981-82 (Paris, 1981/1982), Progr. Math., vol. 38, Birkhäuser Boston, Boston, MA, 1983, pp. 137-151.

[Leh33] D. H. Lehmer, Factorization of certain cyclotomic functions, Ann. of Math. (2) 34 (1933), no. 3, 461-479.

[Mas] D. W. Masser, Lettre à Daniel Bertrand du 17 novembre 1986.

[Mas89] Counting points of small height on elliptic curves, Bull. Soc. Math. France 117 (1989), no. 2, 247-265. 
[Mig78] Maurice Mignotte, Entiers algébriques dont les conjugués sont proches du cercle unité, Séminaire Delange-Pisot-Poitou, 19e année: 1977/78, Théorie des nombres, Fasc. 2, Secrétariat Math., Paris, 1978, pp. Exp. No. 39, 6.

[Rat04] Nicolas Ratazzi, Théorème de Dobrowolski-Laurent pour les extensions abéliennes sur une courbe elliptique à multiplication complexe, Int. Math. Res. Not. (2004), no. 58, 3121-3152.

[Rém05] Gaël Rémond, Intersection de sous-groupes et de sous-variétés. I, Math. Ann. 333 (2005), no. 3, 525-548.

[Rob65] Raphael M. Robinson, Some conjectures about cyclotomic integers, Math. Comp. 19 (1965), 210-217.

[Sil04] Joseph H. Silverman, A lower bound for the canonical height on elliptic curves over abelian extensions, J. Number Theory 104 (2004), no. 2, 353372.

[Smy71] C. J. Smyth, On the product of the conjugates outside the unit circle of an algebraic integer, Bull. London Math. Soc. 3 (1971), 169-175.

[Via03] Evelina Viada, The intersection of a curve with algebraic subgroups in a product of elliptic curves, Ann. Sc. Norm. Super. Pisa Cl. Sci. (5) 2 (2003), no. 1, 47-75.

[Vou96] Paul Voutier, An effective lower bound for the height of algebraic numbers, Acta Arith. 74 (1996), no. 1, 81-95. 